TRANSACTIONS OF THE

AMERICAN MATHEMATICAL SOCIETY

Volume 350, Number 3, March 1998, Pages 1167-1179

S $0002-9947(98) 02127-8$

\title{
THE HOMOLOGICAL DEGREE OF A MODULE
}

\author{
WOLMER V. VASCONCELOS
}

\begin{abstract}
A homological degree of a graded module $M$ is an extension of the usual notion of multiplicity tailored to provide a numerical signature for the module even when $M$ is not Cohen-Macaulay. We construct a degree, hdeg $(M)$, that behaves well under hyperplane sections and the modding out of elements of finite support. When carried out in a local algebra this degree gives a simulacrum of complexity à la Castelnuovo-Mumford's regularity. Several applications for estimating reduction numbers of ideals and predictions on the outcome of Noether normalizations are given.
\end{abstract}

\section{INTRODUCTION}

We construct a homological degree for a graded module $M$ and show how it can be used to produce estimates normally associated to Cohen-Macaulay modules. It leads to a technique to obtain a priori estimates in local algebra.

There are several measures of size of a graded module $M$ over a standard graded ring $S$ : multiplicity, arithmetic and geometric degrees, Castelnuovo-Mumford regularity. There are also related measures of 'good behavior' (or of regularity): depth and projective dimension. Each of these has been used as a complexity, that is as a measure of the cost of extracting information about the module. When used for this purpose, some of these numbers tend to be very large and to regularly overshoot the real cost.

There is a general approach to attaching a degree to a graded module $M$ (or to a module over a local ring): first define a cycle

$$
\operatorname{div}(M)=\sum_{i=1}^{s} \ell_{i}\left[\mathfrak{p}_{i}\right],
$$

where $\ell_{i}$ are rational numbers and $\left[\mathfrak{p}_{i}\right]$ are labels for prime ideals, both derived from $M$, and associating degrees to $\operatorname{div}(M)$. To illustrate, in the case of the arithmetic degree, $\ell_{i}$ will the multiplicity of the prime $\mathfrak{p}_{i}$ as an associated prime of $M$,

$$
\operatorname{adeg}(M)=\sum_{i=1}^{s} \operatorname{mult}_{M}\left(\mathfrak{p}_{i}\right) \operatorname{deg}\left(A / \mathfrak{p}_{i}\right) .
$$

In contrast, the ordinary multiplicity $\operatorname{deg}(M)$ and the geometric degree $\operatorname{gdeg}(M)$ are given by a similar expression in which only the primes $\mathfrak{p}_{i}$ of $\operatorname{dimension} \operatorname{dim}(M)$ are

Received by the editors June 3, 1996.

1991 Mathematics Subject Classification. Primary 13D40; Secondary 13D45, 13P10.

Key words and phrases. Arithmetic degree, Castelnuovo-Mumford regularity, geometric degree, Gorenstein ring, homological degree, reduction number.

The author was partially supported by the NSF. 
taken in $\operatorname{deg}(M)$, and only the minimal primes in the support of $M$ are considered for $\operatorname{gdeg}(M)$.

Our guide in defining new degrees is the following rule: It must generalize the ordinary length $\ell(\cdot)$ and behave well under generic hyperplane section. In the case of $\operatorname{adeg}(M)$, one has for a regular hyperplane section $\operatorname{adeg}(M) \leq \operatorname{adeg}(M / h M)$, often with strict inequality. Technically, what is needed is another notion of degree, $\operatorname{Deg}(\cdot)$, which reduces to the usual multiplicity in the Cohen-Macaulay case, with the following two additional requirements:

(i) If $L=\Gamma_{\mathfrak{m}}(M)$ is the submodule of elements of finite support of $M$ and $\bar{M}=M / L$, then

$$
\operatorname{Deg}(M)=\operatorname{Deg}(\bar{M})+\ell(L) .
$$

(ii) If $h \in S$ is a regular, generic hyperplane section on $M$, then

$$
\operatorname{Deg}(M) \geq \operatorname{Deg}(M / h M) .
$$

There may exist many definitions for such degrees. One advantage is that each would afford a priori estimates for bounds one normally finds in Cohen-Macaulay modules as expressed by the ordinary multiplicity. Unfortunately, if $M$ is not Cohen-Macaulay, $\operatorname{deg}(M)$ leaks all over as a predictor of properties of $M$, and in a smaller measure so $\operatorname{do} \operatorname{adeg}(M)$ and the Castelnuovo-Mumford regularity $\operatorname{reg}(M)$ as well.

Let us now outline the contents. Section 2 recalls the definitions of all major degrees we discuss. Two features of the degrees are emphasized: their behavior under hyperplane sections and their explicit computation by Computer Algebra Systems such as CoCoA ([4]) or Macaulay ([2]). The main device introduced is the notion of a homological degree of a module $M$. It is supported on the prime ideals associated to $M$, but at all levels of its local cohomology. Thus (now we assume that $S$ is a Gorenstein ring) the prime ideals

$$
\bigcup_{i \geq 0} \operatorname{Ass}\left(\operatorname{Ext}_{S}^{i}(M, S)\right) \backslash \operatorname{Ass}(M)
$$

will play a significant role in the construction. These primes themselves will be called the hidden associated primes of $M$. (Of course, this will lead to the notion of well hidden associated prime!)

The degree introduced here has the recursive form: If $S$ is a ring of polynomials in $d$ indeterminates over a field and $M$ is a graded module, then

$$
\operatorname{Deg}(M)=\operatorname{deg}(M)+\sum_{i=1}^{d} c_{i} \cdot \operatorname{Deg}\left(\operatorname{Ext}_{S}^{i}(M, S)\right),
$$

where the $c_{i}$ 's are binomial coefficients chosen to accommodate the good behavior of $\operatorname{Deg}(\cdot)$ under hyperplane sections. It is reminiscent of formulas that occur in the theory of Buchsbaum modules. It suffers from some deficiencies, the most glaring being that $\operatorname{Deg}(M)$ and $\operatorname{Deg}(M / h M)$ may differ when $h$ is regular. Nevertheless, our main result (Theorem 2.13) asserts that for a suitably defined notion of generic hyperplane section, the hard inequality (2) holds. It will suffice for all our applications.

The last section shows how a sharp degree function $\operatorname{Deg}(\cdot)$ gives an extension of the elegant estimates of Sally and others (see [9]) on the multiplicity and reduction number of tangent cones arising from Cohen-Macaulay rings to general local rings. 
More precisely, for a local ring $(R, \mathfrak{m})$ which is a homomorphic image of a Gorenstein ring (e.g. all geometric local rings), we define the notion of homological multiplicity by setting $e_{h}(R)=\operatorname{Deg}(R)$. Most of the estimates known to hold in CohenMacaulay rings on polynomial bounds on number of generators of primary ideals are then extended to the rings here. In some sense, $e_{h}(R)$ plays a role similar to the Castelnuovo-Mumford regularity in graded rings.

\section{The Degrees of A Module}

The algebras we will consider are of two kinds: (i) Noetherian local rings $(R, \mathfrak{m})$, or (ii) finitely generated, positively graded algebras over an Artinian local $A_{0}$,

$$
A=A_{0}+A_{1}+A_{2}+\cdots=A_{0}+A_{+},
$$

where $A_{i}$ denotes the space of homogeneous elements of degree $i$. We further assume that $A$ is generated by its 1 -forms, $A=A_{0}\left[A_{1}\right]$, in which case $A_{i}=A_{1}{ }^{i}$. Such algebras are said to be standard.

The notation throughout is that of [3], particularly where it concerns facts of local cohomology.

Multiplicity. Let $(R, \mathfrak{m})$ be a local ring and let $M$ be a finitely generated $R$-module. The Hilbert-Samuel function of $M$ is the length function

$$
H S_{M}: n \mapsto \ell\left(M / \mathfrak{m}^{n} M\right),
$$

which for $n \gg 0$ is given by a polynomial:

$$
H S_{M}(n)=\frac{\operatorname{deg}(M)}{d !} n^{d}+\text { lower terms. }
$$

If $M$ is a graded module over a standard algebra $A$, these functions are routinely computed by programs such as $C o C o A$ or Macaulay ([4], [2]).

Definition 2.1. The integer $\operatorname{deg}(M)$ is the multiplicity of the module $M$.

Geometric and arithmetic degrees. For a wealth of details and applications of these degrees, we refer to [11]. For notation, if $I$ is an ideal of a $\operatorname{ring} R$ and $M$ is an $R$-module, $\Gamma_{I}(M)$ is the submodule of $M$ of all elements with support in the closed set $V(I)$.

Definition 2.2. Let $R$ be a Noetherian ring and $M$ a finitely generated $R$-module. For a prime ideal $\mathfrak{p} \subset R$, the integer

$$
\operatorname{mult}_{M}(\mathfrak{p})=\ell\left(\Gamma_{\mathfrak{p}}\left(M_{\mathfrak{p}}\right)\right)
$$

is the length multiplicity of $\mathfrak{p}$ with respect to $M$.

This number $\operatorname{mult}_{M}(\mathfrak{p})$, which vanishes if $\mathfrak{p}$ is not an associated prime of $M$, is a measure of the contribution of $\mathfrak{p}$ to the primary decomposition of the null submodule of $M$. It is not usually accessible through computation, although certain linear combinations can be computed.

Several authors have shown the significance of the following weighted linear combination of length multiplicities ([1], [6], [11]):

Definition 2.3. The arithmetic degree of $M$ is the integer

$$
\operatorname{adeg}(M)=\sum_{\mathfrak{p} \in \operatorname{Ass}(M)} \operatorname{mult}_{M}(\mathfrak{p}) \cdot \operatorname{deg} A / \mathfrak{p} .
$$


The definition applies to more general modules, not just graded modules, although its main use is for graded modules over a standard algebra $A$. If all the associated primes of $M$ have the same dimension, then $\operatorname{adeg}(M)$ is just the multiplicity $\operatorname{deg}(M)$ of $M$, which is obtained from its Hilbert polynomial.

This sum can be determined without the knowledge of primary decomposition ([16]). Taking a Noether normalization of $A$, one has:

Proposition 2.4. Let $M$ be a finitely generated graded module over the ring $S=$ $k\left[x_{1}, \ldots, x_{n}\right]$. Then

$$
\operatorname{adeg}(M)=\sum_{i=0}^{n} \operatorname{deg}\left(\operatorname{Ext}_{S}^{i}\left(\operatorname{Ext}_{S}^{i}(M, S), S\right)\right) .
$$

The geometric degree $\operatorname{gdeg}(M)$ is the partial sum from (3), corresponding to the minimal primes of $\operatorname{Ass}(M)$.

In [16] we discussed a relationship between $\operatorname{adeg}(A)$ and certain qualitative aspects on carrying out Noether normalization on $A$. Unfortunately this degree does not always behave well under hyperplane sections. The following, obtained by a straightforward computation, is valuable in locating the gaps to be patched (see also [1], [7]).

Proposition 2.5. Let $A$ be a standard graded algebra, let $M$ be a graded algebra and let $h \in A_{1}$ be a regular element on $M$. Then

$$
\operatorname{adeg}(M / h M) \geq \operatorname{adeg}(M) .
$$

Example 2.6. A simple example where strict inequality holds is the ideal of two disjoint lines in $\mathbb{P}^{3}: A=k[x, y, z, w] / I$, with $I=(x, y) \cap(z, w)$ and $h=x+z$; then $\operatorname{adeg}(A)=2<3=\operatorname{adeg}(A / h A)$.

Castelnuovo-Mumford regularity. Let $k$ be a field, $S=k\left[x_{1}, \ldots, x_{m}\right]$ a polynomial ring over $k, R=S / I$ a homogeneous $k$-algebra, and $M$ a finitely generated graded $R$-module. Then $M$, as an $S$-module, admits a finite graded free resolution:

$$
0 \rightarrow \bigoplus_{j} S(-j)^{b_{p j}} \longrightarrow \cdots \longrightarrow \bigoplus_{j} S(-j)^{b_{0 j}} \longrightarrow M \rightarrow 0 .
$$

Definition 2.7. The Castelnuovo-Mumford regularity of $M$ is the integer

$$
\operatorname{reg} M=\max \left\{j-i: b_{i j} \neq 0\right\} .
$$

In other words, $\operatorname{reg} M=\max \left\{\alpha_{+}\left(\operatorname{Tor}_{i}^{S}(M, k)\right)-i: i \in \mathbb{Z}\right\}$, where for a graded module $N$ with $N_{j}=0$ for large $j$ we set $\alpha_{+}(N)=\max \left\{j: N_{j} \neq 0\right\}$. Let $q$ be an integer. The module $M$ is called q-regular if $q \geq \operatorname{reg}(M)$, equivalently, if $\operatorname{Tor}_{i}^{S}(M, k)_{j+i}=0$ for all $i$ and all $j>q$.

The homological degree of a module. We now construct a homological degree for a graded module. There is an immediate extension of this notion to modules over local rings which are homomorphic images of a Gorenstein ring.

Definition 2.8. Let $M$ be a finitely generated graded module over the graded algebra $A$ and let $S$ be a Gorenstein graded algebra mapping onto $A$, with maximal graded ideal $\mathfrak{m}$. Assume that $\operatorname{dim} S=r, \operatorname{dim} M=d$. The homological degree of $M$ is the integer

$$
\operatorname{hdeg}(M)=\operatorname{deg}(M)+\sum_{i=r-d+1}^{r}\left(\begin{array}{c}
d-1 \\
i-r+d-1
\end{array}\right) \cdot \operatorname{hdeg}\left(\operatorname{Ext}_{S}^{i}(M, S)\right) .
$$


This expression becomes more compact when $\operatorname{dim} M=\operatorname{dim} S=d>0$ :

$$
\operatorname{hdeg}(M)=\operatorname{deg}(M)+\sum_{i=1}^{d}\left(\begin{array}{c}
d-1 \\
i-1
\end{array}\right) \cdot \operatorname{hdeg}\left(\operatorname{Ext}_{S}^{i}(M, S)\right) .
$$

Since the action of $S$ on $M$ passes through the action of $A$ on $M$, it is not difficult to show that $\operatorname{hdeg}(M)$ is independent of $S$. This also follows from the local duality theorem ([3]).

Example 2.9. We illustrate how this degree compares with the degrees of the previous section with the following example of Bernd Ulrich. Let $A=k[x, y, u, v] / I$, with $I=\left((x, y)^{2}, x u^{t}+y v^{t}\right)$. If $t \geq 3$,

$$
\operatorname{deg}(A)=\operatorname{adeg}(A)=2<\operatorname{reg}(A)=t<\operatorname{hdeg}(A)=2+t^{2} .
$$

Remark 2.10. If $M$ is a module that is Cohen-Macaulay on the punctured spectrum, this degree becomes

$$
\operatorname{hdeg}(M)=\operatorname{deg}(M)+\sum_{i=0}^{d-1}\left(\begin{array}{c}
d-1 \\
i
\end{array}\right) \cdot \ell\left(H_{\mathfrak{m}}^{i}(M)\right) .
$$

Note that the binomial term is the Stückrad-Vogel invariant in the theory of Buchsbaum modules ([10, Proposition 2.6]).

The equality (1) is immediate (see also Proposition 2.14), but (2) is hard. We phrase a broader version of it as:

Conjecture 2.11. Let $M$ be a graded module and let $h$ be a regular hyperplane section. Then

$$
\operatorname{hdeg}(M) \geq \operatorname{hdeg}(M / h M) .
$$

We shall deal with this question by identifying a specific family of hyperplane sections.

Generic hyperplane section. Let $S$ be a Gorenstein standard graded ring with infinite residue field, and let $M$ be a finitely generated graded module over $S$. We recall that a superficial element of order $r$ for $M$ is an element $z \in S_{r}$ such that $0:{ }_{M} z$ is a submodule of $M$ of finite length.

Definition 2.12. A special hyperplane section of $M$ is an element $h \in S_{1}$ that is superficial for all the iterated Exts

$$
M_{i_{1}, i_{2}, \ldots, i_{p}}=\operatorname{Ext}_{S}^{i_{1}}\left(\operatorname{Ext}_{S}^{i_{2}}\left(\cdots\left(\operatorname{Ext}_{S}^{i_{p-1}}\left(\operatorname{Ext}_{S}^{i_{p}}(M, S), S\right), \cdots, S\right)\right)\right),
$$

and all sequences of integers $i_{1} \geq i_{2} \geq \cdots \geq i_{p} \geq 0$.

By local duality it follows that, up to shifts in grading, there are only finitely many such modules. Actually, it is enough to consider those sequences in which $i_{1} \leq \operatorname{dim} S$ and $p \leq 2 \operatorname{dim} S$, which ensures the existence of such 1 -forms as $h$. It is clear that this property holds for generic hyperplane sections.

The following result proves a case of the conjecture which will suffice for all our applications.

Theorem 2.13. Let $S$ be a standard Gorenstein graded algebra and let $M$ be a finitely generated graded module of depth at least 1 . If $h \in S$ is a special generic hyperplane section on $M$, then

$$
\operatorname{hdeg}(M) \geq \operatorname{hdeg}(M / h M) .
$$


Proof. We will require several technical reductions. We assume that $h$ is a regular, generic hyperplane section for the module $M$ which is regular on $S$. We also assume that $\operatorname{dim} M=\operatorname{dim} S=d$, and derive several exact sequences from

$$
0 \rightarrow M \stackrel{h}{\longrightarrow} M \longrightarrow N \rightarrow 0 \text {. }
$$

For simplicity, we write $M_{i}=\operatorname{Ext}_{S}^{i}(M, S)$, and $N_{i}=\operatorname{Ext}_{S}^{i+1}(N, S)$ in the case of $N$. (The latter because $N$ is a module of dimension $\operatorname{dim} S-1$, and the module $\left.N_{i}=\operatorname{Ext}_{S /(h)}^{i}(N, S /(h)).\right)$

Using this notation, in view of the binomial coefficients in the definition of $\operatorname{hdeg}(\cdot)$, it will be enough to show that

$$
\operatorname{hdeg}\left(N_{i}\right) \leq \operatorname{hdeg}\left(M_{i}\right)+\operatorname{hdeg}\left(M_{i+1}\right) \text {, for } i \geq 1 \text {. }
$$

The sequence (9) gives rises to the long sequence of cohomology

$$
\begin{gathered}
0 \rightarrow M_{0} \longrightarrow M_{0} \longrightarrow N_{0} \longrightarrow M_{1} \longrightarrow M_{1} \longrightarrow N_{1} \longrightarrow M_{2} \longrightarrow \cdots \\
\cdots \longrightarrow M_{d-2} \longrightarrow M_{d-2} \longrightarrow N_{d-2} \longrightarrow M_{d-1} \longrightarrow M_{d-1} \longrightarrow N_{d-1} \rightarrow 0,
\end{gathered}
$$

which are broken up into shorter exact sequences as follows:

$$
\begin{aligned}
0 & \rightarrow L_{i} \longrightarrow M_{i} \longrightarrow \widetilde{M}_{i} \rightarrow 0, \\
0 & \rightarrow \widetilde{M}_{i} \longrightarrow M_{i} \longrightarrow G_{i} \rightarrow 0, \\
0 & \rightarrow G_{i} \longrightarrow N_{i} \longrightarrow L_{i+1} \rightarrow 0
\end{aligned}
$$

We note that all $L_{i}$ have finite length from the condition on $h$. For $i=0$, we have the usual relation, $\operatorname{deg}(M)=\operatorname{deg}(N)$. In case $\widetilde{M}_{i}$ has finite length, then $M_{i}, G_{i}$ and $N_{i}$ have finite length, and

$$
\begin{aligned}
\operatorname{hdeg}\left(N_{i}\right) & =\ell\left(N_{i}\right)=\ell\left(G_{i}\right)+\ell\left(L_{i+1}\right) \\
& \leq \operatorname{hdeg}\left(M_{i}\right)+\operatorname{hdeg}\left(M_{i+1}\right) .
\end{aligned}
$$

It is a similar relation that we want to establish for all other cases.

Proposition 2.14. Let $S$ be a Gorenstein graded algebra and let

$$
0 \rightarrow A \longrightarrow B \longrightarrow C \rightarrow 0
$$

be an exact sequence of graded modules. Then

(a) If $A$ is a module of finite length, then

$$
\operatorname{hdeg}(B)=\operatorname{hdeg}(A)+\operatorname{hdeg}(C) .
$$

(b) If $C$ is a module of finite length, then

$$
\operatorname{hdeg}(B) \leq \operatorname{hdeg}(A)+\operatorname{hdeg}(C) .
$$

Proof. They are both clear if $B$ is a module of finite length, so we assume $\operatorname{dim} B=$ $d \geq 1$.

(a): This is immediate, since $\operatorname{deg}(B)=\operatorname{deg}(C)$ and the cohomology sequence gives

$$
\begin{aligned}
\operatorname{Ext}_{S}^{i}(B, S) & =\operatorname{Ext}_{S}^{i}(C, S), 1 \leq i \leq d-1, \\
\ell\left(\operatorname{Ext}_{S}^{d}(B, S)\right) & =\ell\left(\operatorname{Ext}_{S}^{d}(A, S)\right)+\ell\left(\operatorname{Ext}_{S}^{d}(C, S)\right) .
\end{aligned}
$$

(b): Similarly we have

$$
\operatorname{Ext}_{S}^{i}(B, S)=\operatorname{Ext}_{S}^{i}(A, S), 1 \leq i<d-1,
$$


and the exact sequence

$$
0 \rightarrow \operatorname{Ext}_{S}^{d-1}(B, S) \rightarrow \operatorname{Ext}_{S}^{d-1}(A, S) \rightarrow \operatorname{Ext}_{S}^{d}(C, S) \rightarrow \operatorname{Ext}_{S}^{d}(B, S) \rightarrow \operatorname{Ext}_{S}^{d}(A, S) \rightarrow 0 .
$$

If $\operatorname{Ext}_{S}^{d-1}(A, S)$ has finite length,

$$
\begin{gathered}
\operatorname{hdeg}\left(\operatorname{Ext}_{S}^{d-1}(B, S)\right) \leq \operatorname{hdeg}\left(\operatorname{Ext}_{S}^{d-1}(A, S)\right), \\
\operatorname{hdeg}\left(\operatorname{Ext}_{S}^{d}(B, S)\right) \leq \operatorname{hdeg}\left(\operatorname{Ext}_{S}^{d}(A, S)\right)+\operatorname{hdeg}\left(\operatorname{Ext}_{S}^{d}(, S)\right) .
\end{gathered}
$$

Otherwise, $\operatorname{dim} \operatorname{Ext}_{S}^{d-1}(A, S)=1$, and

$$
\operatorname{hdeg}\left(\operatorname{Ext}_{S}^{d-1}(A, S)\right)=\operatorname{deg}\left(\operatorname{Ext}_{S}^{d-1}(A, S)\right)+\ell\left(\Gamma_{\mathfrak{m}}\left(\operatorname{Ext}_{S}^{d-1}(A, S)\right)\right) .
$$

Since we also have

$$
\begin{gathered}
\operatorname{deg}\left(\operatorname{Ext}_{S}^{d-1}(B, S)\right)=\operatorname{deg}\left(\operatorname{Ext}_{S}^{d-1}(A, S)\right), \\
\ell\left(\Gamma_{\mathfrak{m}}\left(\operatorname{Ext}_{S}^{d-1}(B, S)\right)\right) \leq \ell\left(\Gamma_{\mathfrak{m}}\left(\operatorname{Ext}_{S}^{d-1}(A, S)\right)\right),
\end{gathered}
$$

we again obtain the stated bound.

Suppose $\operatorname{dim} \widetilde{M}_{i} \geq 1$. From Proposition $2.14(\mathrm{~b})$ we have

$$
\operatorname{hdeg}\left(N_{i}\right) \leq \operatorname{hdeg}\left(G_{i}\right)+\ell\left(L_{i+1}\right) .
$$

We must now relate $\operatorname{hdeg}\left(G_{i}\right)$ to $\operatorname{deg}\left(M_{i}\right)$. Apply the functor $\Gamma_{\mathfrak{m}}(\cdot)$ to the sequence (12) and consider the commutative diagram

$$
\begin{aligned}
& 0 \quad \rightarrow \quad \widetilde{M}_{i} \quad \longrightarrow \quad M_{i} \quad \longrightarrow \quad G_{i} \quad \rightarrow 0 \\
& 0 \rightarrow \Gamma_{\mathfrak{m}}\left(\widetilde{M}_{i}\right) \longrightarrow \Gamma_{\mathfrak{m}}\left(M_{i}\right) \longrightarrow \Gamma_{\mathfrak{m}}\left(G_{i}\right)
\end{aligned}
$$

in which we denote by $H_{i}$ the image of

$$
\Gamma_{\mathfrak{m}}\left(M_{i}\right) \longrightarrow \Gamma_{\mathfrak{m}}\left(G_{i}\right)
$$

Through the snake lemma, we obtain the exact sequence

$$
0 \rightarrow \widetilde{M}_{i} / \Gamma_{\mathfrak{m}}\left(\widetilde{M}_{i}\right) \stackrel{\alpha}{\longrightarrow} M_{i} / \Gamma_{\mathfrak{m}}\left(M_{i}\right) \longrightarrow G_{i} / H_{i} \rightarrow 0
$$

Furthermore, from (11) there is a natural isomorphism,

$$
\beta: M_{i} / \Gamma_{\mathfrak{m}}\left(M_{i}\right) \simeq \widetilde{M}_{i} / \Gamma_{\mathfrak{m}}\left(\widetilde{M}_{i}\right)
$$

while from (12) there is a natural injection

$$
\widetilde{M}_{i} / \Gamma_{\mathfrak{m}}\left(\widetilde{M}_{i}\right) \hookrightarrow M_{i} / \Gamma_{\mathfrak{m}}\left(M_{i}\right),
$$

whose composite with $\beta$ is induced by multiplication by $h$ on $M_{i} / \Gamma_{\mathfrak{m}}\left(M_{i}\right)$. We may thus replace $\widetilde{M}_{i} / \Gamma_{\mathfrak{m}}\left(\widetilde{M}_{i}\right)$ by $M_{i} / \Gamma_{\mathfrak{m}}\left(M_{i}\right)$ in (15) and take $\alpha$ as multiplication by $h$ :

$$
0 \rightarrow M_{i} / \Gamma_{\mathfrak{m}}\left(M_{i}\right) \stackrel{h}{\longrightarrow} M_{i} / \Gamma_{\mathfrak{m}}\left(M_{i}\right) \longrightarrow G_{i} / H_{i} \rightarrow 0
$$

Observe that since

$$
\operatorname{Ext}_{S}^{j}\left(M_{i} / \Gamma_{\mathfrak{m}}\left(M_{i}\right), S\right)=\operatorname{Ext}_{S}^{j}\left(M_{i}, S\right), j<\operatorname{dim} S,
$$

$h$ is still a regular, generic hyperplane section for $M_{i} / \Gamma_{\mathfrak{m}}\left(M_{i}\right)$. By induction on the dimension of the module, we have

$$
\operatorname{hdeg}\left(M_{i} / \Gamma_{\mathfrak{m}}\left(M_{i}\right)\right) \geq \operatorname{hdeg}\left(G_{i} / H_{i}\right) .
$$

Now from Proposition 2.14(a), we have

$$
\operatorname{hdeg}\left(G_{i}\right)=\operatorname{hdeg}\left(G_{i} / H_{i}\right)+\ell\left(H_{i}\right) .
$$


Since these summands are bounded by $\operatorname{hdeg}\left(M_{i} / \Gamma_{\mathfrak{m}}\left(M_{i}\right)\right)$ and $\ell\left(\Gamma_{\mathfrak{m}}\left(M_{i}\right)\right)$, respectively (in fact, $\ell\left(H_{i}\right)=\ell\left(L_{i}\right)$ ), we have

$$
\operatorname{hdeg}\left(G_{i}\right) \leq \operatorname{hdeg}\left(M_{i} / \Gamma_{\mathfrak{m}}\left(M_{i}\right)\right)+\ell\left(\Gamma_{\mathfrak{m}}\left(M_{i}\right)\right)=\operatorname{hdeg}\left(M_{i}\right),
$$

the last equality by Proposition 2.14(a) again. Finally, taking this estimate into (14) we get

$$
\begin{aligned}
\operatorname{hdeg}\left(N_{i}\right) & \leq \operatorname{hdeg}\left(G_{i}\right)+\ell\left(L_{i+1}\right) \\
& \leq \operatorname{hdeg}\left(M_{i}\right)+\operatorname{hdeg}\left(M_{i+1}\right),
\end{aligned}
$$

to establish the claim.

Remark 2.15. That equality does not always hold is shown by the following example. Let $R=k[x, y]$ and $M=(x, y)^{2}$. Then $\operatorname{hdeg}(M)=4$, but for any hyperplane section $h, \operatorname{hdeg}(M / h M)=3$. To get an example of a ring one takes the idealization of $M$.

We shall need the following consequence of the technical details in the proof:

Corollary 2.16. Let $M$ and $h$ be as above and let $r$ be a positive integer. Then

$$
r \cdot \operatorname{hdeg}(M) \geq \operatorname{hdeg}\left(M / h^{r} M\right) .
$$

Proof. We will again argue by induction on the dimension of $M$, keeping the notation above on the exact sequence

$$
0 \rightarrow M \stackrel{h^{r}}{\longrightarrow} M \longrightarrow N \rightarrow 0 .
$$

To begin with, we always have $\operatorname{deg}\left(M / h^{r} M\right)=r \cdot \operatorname{deg}(M)$. In all of the previous proof there are just two places where $h$, instead of $h^{r}$, has significance. First, from (17) we have

$$
\operatorname{hdeg}\left(N_{i}\right) \leq \operatorname{hdeg}\left(G_{i}\right)+\ell\left(\widetilde{L}_{i+1}\right), i \geq 1 .
$$

Note that $\widetilde{L}_{i}$ denotes the kernel of the multiplication by $h^{r}$ on $M_{i}$; by induction on $r$ and the snake lemma, $\ell\left(\widetilde{L}_{i+1}\right) \leq r \cdot \ell\left(L_{i+1}\right)$. One also has

$$
\operatorname{hdeg}\left(G_{i}\right)=\operatorname{hdeg}\left(G_{i} / H_{i}\right)+\ell\left(H_{i}\right),
$$

while from (15) and induction, we have that

$$
\begin{aligned}
\operatorname{hdeg}\left(G_{i} / H_{i}\right) & \leq r \cdot \operatorname{hdeg}\left(M_{i} / \Gamma_{\mathfrak{m}}\left(M_{i}\right)\right) \\
& =r \cdot\left(\operatorname{hdeg}\left(M_{i}\right)-\ell\left(\Gamma_{\mathfrak{m}}\left(M_{i}\right)\right)\right) .
\end{aligned}
$$

Finally, noting that $H_{i}$ is a homomorphic image of $\Gamma_{\mathfrak{m}}\left(M_{i}\right)$ (as remarked above, tracing through, it holds that $\ell\left(H_{i}\right)=\ell\left(\widetilde{L}_{i}\right)$, which is in any event bounded by $\ell\left(\Gamma_{\mathfrak{m}}\left(M_{i}\right)\right)$ since $h$, and therefore $h^{r}$, is also a superficial element).

Adding all pieces together, we get

$$
\begin{aligned}
\operatorname{hdeg}\left(N_{i}\right) & \leq r \cdot \operatorname{hdeg}\left(M_{i}\right)+r \cdot \ell\left(L_{i+1}\right) \\
& -r \cdot \ell\left(\Gamma_{\mathfrak{m}}\left(M_{i}\right)\right)+\ell\left(H_{i}\right),
\end{aligned}
$$

which on dropping the second line and replacing $\ell\left(L_{i+1}\right)$ by hdeg $\left(M_{i+1}\right)$ gives

$$
\operatorname{hdeg}\left(N_{i}\right) \leq r \cdot\left(\operatorname{hdeg}\left(M_{i}\right)+\operatorname{hdeg}\left(M_{i+1}\right)\right),
$$

and we finish as in the theorem. 
Remark 2.17. The arguments show that in trying to plug the leaks that occur in the formula for the arithmetic degree under hyperplane section

$$
\operatorname{adeg}(M) \leq \operatorname{adeg}(M / h M),
$$

we may have gone too far in establishing

$$
\operatorname{hdeg}(M) \geq \operatorname{hdeg}(M / h M) .
$$

Perhaps, somewhere in between, there exists a degree function $\operatorname{Deg}(M)$ that gives equality. Although $\mathrm{hdeg}(\cdot)$ fulfills the key requisite of giving a priori estimates, the proofs show several places when degree counts may have been overstated.

\section{Complexity BOUNDS IN LOCAL RINGS}

Let $(R, \mathfrak{m})$ be a Noetherian local ring and let $I \subset R$ be an ideal. The study of certain algebras constructed with $I$, such as its Rees algebra $R[I t]$ with its special fiber $F(I)=R[I t] \otimes(R / \mathfrak{m})$, provides a setting for application of the methods of the two preceding sections. Here we focus on $F(I)$, which is an ordinary standard graded algebra, and attempt to connect arithmetical properties of $I$ to the complexity bounds of $F(I)$.

Homological multiplicity. We begin by observing that it still makes sense to define the arithmetic degree, $\operatorname{adeg}(R)$, of the local ring $R$ : In the formula (3), the multiplicity $\operatorname{deg}(R / \mathfrak{p})$ of the local ring $R / \mathfrak{p}$ replaces $\operatorname{deg}(A / \mathfrak{p})$. The geometric degree, $\operatorname{gdeg}(R)$, is defined similarly, but $\operatorname{reg}(A)$ has no obvious extension.

In order to define hdeg $(R)$ we must assume that $R$ is the homomorphic image of a Gorenstein ring. Since the definition of this degree seems to depend on the presentation $S \mapsto R$, we simply take the minimum of such values. In some cases, for example, if $R$ is complete, $\operatorname{hdeg}(R)$ is by local duality independent of $S$. In order to define $\operatorname{hdeg}(R)$ we must assume that $R$ is the homomorphic image of a Gorenstein ring. In some cases, for example, if $R$ is complete, $\operatorname{hdeg}(R)$ is by local duality independent of $S$.

Definition 3.1. Let $R$ be a local ring that is the homomorphic image of a Gorenstein ring. The homological multiplicity is the integer $e_{h}(R)=\operatorname{hdeg}(R)$.

It would be of interest to construct degree functions $\operatorname{Deg}(\cdot)$, sharper than $h d e g(\cdot)$, but still satisfying the following two conditions:

(i) If $L=\Gamma_{\mathfrak{m}}(M)$ is the submodule of elements of finite support of $M$ and $\bar{M}=M / L$, then

$$
\operatorname{Deg}(M)=\operatorname{Deg}(\bar{M})+\ell(L) .
$$

(ii) If $h \in S$ is a regular hyperplane section on $M$, then

$$
\operatorname{Deg}(M) \geq \operatorname{Deg}(M / h M) \text {. }
$$

In the previous section, we saw that $\operatorname{hdeg}(M)$ does have this attribute, but it may be overstated when it deals with condition (ii). Getting equality would be considerably better.

The relationship between these functions and the minimal number of generator functions $\nu_{R}(\cdot)$ is easy to express. 
Proposition 3.2. Let $\operatorname{Deg}(\cdot)$ be a degree function satisfying $(i)$ and $(i i)$ above. Then for any finitely generated module $M$,

$$
\nu_{R}(M) \leq \operatorname{Deg}(M)
$$

Proof. One inducts on $d=\operatorname{dim}(M)$. If $d=0, \operatorname{Deg}(M)=\ell(M) \geq \nu_{R}(M)$. In case $d>0$, if $L=\Gamma_{\mathfrak{m}}(M) \neq 0$, the condition $(i)$ implies that it will be enough to prove the assertion for $N=M / L$, a module of depth $>0$. Let $h \in \mathfrak{m}$ be a generic hyperplane section on $M$; from (ii)

$$
\operatorname{Deg}(N) \geq \operatorname{Deg}(N / h N) \geq \nu_{R}(N / h N)=\nu_{R}(N)
$$

the second inequality by the induction hypothesis and the last equality by Nakayama's lemma.

Corollary 3.3. Let $S$ be a Gorenstein local ring (or a standard Gorenstein graded algebra) and let $M$ be a finitely generated (resp. finitely generated, graded) $S-$ module. Then

$$
\operatorname{hdeg}(M) \geq \nu_{S}(M) .
$$

Bounding the number of generators of ideals with multiplicities. The notion of homological degree of to estimate the number of generators of an ideal in terms of various degrees of $R$ and of $R / I$.

Example 3.4. (i) Let $R$ be a Cohen-Macaulay local ring of dimension $d$ and let $I$ be a Cohen-Macaulay ideal of height $g$. Suppose $S \rightarrow R$ is a Gorenstein presentation and $\operatorname{dim} S=d$.

The exact sequence

$$
0 \rightarrow I \longrightarrow R \longrightarrow R / I \rightarrow 0
$$

permits a comparison between $\operatorname{hdeg}(R / I)=\operatorname{deg}(R / I)$ and $\operatorname{hdeg}(I)$.

If $g \leq 1$, then $I$ is Cohen-Macaulay and

$$
\operatorname{hdeg}(I)=\operatorname{deg}(I)= \begin{cases}\operatorname{deg}(R)-\operatorname{deg}(R / I), & \text { if } g=0, \\ \operatorname{deg}(R), & \text { if } g=1 .\end{cases}
$$

If $g \geq 2$, then $I$ is not a Cohen-Macaulay module and

$$
\operatorname{Ext}_{S}^{g-1}(I, S) \simeq \operatorname{Ext}_{S}^{g}(R / I, S) .
$$

We then have

$$
\operatorname{hdeg}(I)=\operatorname{deg}(R)+\left(\begin{array}{l}
d-1 \\
g-2
\end{array}\right) \operatorname{deg}(R / I) .
$$

Since in general we have $\nu(I) \leq \operatorname{hdeg}(I)$, this gives the estimate

$$
\nu(I) \leq \operatorname{deg}(R)+\left(\begin{array}{l}
d-1 \\
g-2
\end{array}\right) \operatorname{deg}(R / I) .
$$

For the purpose of finding $\nu(I)$ this can be considerably improved, as follows. First we make a reduction to the case of an infinite residue field in the usual manner. If $d>g$, we may reduce the exact sequence (18) modulo a superficial element $h$ for both $R$ and $R / I$. The sequence (18) gives rise to

$$
0 \rightarrow I^{\prime} \longrightarrow R^{\prime} \longrightarrow R^{\prime} / I^{\prime} \rightarrow 0,
$$


in which $I^{\prime}=I / h I$ (and therefore $\nu(I)=\nu\left(I^{\prime}\right)$ ) and the multiplicities are retained. Repeating, we end up in the case $d=g$,

$$
\nu(I) \leq \operatorname{deg}(R)+\left(\begin{array}{l}
g-1 \\
g-2
\end{array}\right) \operatorname{deg}(R / I)=\operatorname{deg}(R)+(g-1) \operatorname{deg}(R / I) .
$$

This is however not yet as sharp as the estimate of [14].

(ii) It is not difficult to see that if $R$ is a local ring which is the homomorphic image of a Gorenstein ring and $I$ is an ideal of $R$, of dimension $d-g$, and $R / I$ is Cohen-Macaulay, then the analogous estimate for the number of generators of $I$ holds,

$$
\nu(I) \leq \operatorname{hdeg}(R)+(g-1) \operatorname{deg}(R / I) .
$$

(iii) If $R / I$ is not Cohen-Macaulay the approach still yields some estimates. Consider the case in which $R$ is Cohen-Macaulay of dimension $d$ and $\operatorname{depth}(R / I) \geq$ $d-g-1$. In a similar manner as above, we have

$$
\nu(I) \leq \operatorname{deg}(R)+\left(\begin{array}{l}
g \\
2
\end{array}\right) \cdot \operatorname{deg}(R / I)+g \cdot \ell\left(\Gamma_{\mathfrak{m}}(R /(I, \mathbf{x}))\right),
$$

where $\mathbf{x}=x_{1}, \ldots, x_{d-g-1}$ is a regular, superficial sequence for both $R$ and $R / I$.

Several bounds on the multiplicity of graded algebras such as $F(I)$, arising from Cohen-Macaulay rings, were obtained by J. Sally and others ([8], [9], [12], [13]). We give one of her spare proofs, where $e_{h}(R)$ replaces the ordinary multiplicity in the case of Cohen-Macaulay ring.

Theorem 3.5. Let $(R, \mathfrak{m})$ be a local ring of dimension $d>0$, which is the homomorphic image of a Gorenstein ring. Let $I$ be an $\mathfrak{m}$-primary ideal and $s$ the index of nilpotency of $R / I$. Then

$$
\nu(I) \leq s^{d-1} \cdot e_{h}(R)+d-1 .
$$

Proof. We may assume that the residue field of $R$ is infinite, as the passage from $R$ to $R[T]_{\mathfrak{m} R[T]}$ can only improve $e_{h}(R)$.

The proof is by induction on $d$. Suppose $(R, \mathfrak{m})$ is a local ring of dimension 1 , and $I$ is $\mathfrak{m}$-primary. If $L=\Gamma_{\mathfrak{m}}(R)$, the exact sequence

$$
0 \rightarrow I \cap L \longrightarrow I \longrightarrow I \bar{R} \rightarrow 0,
$$

where $\bar{R}=R / L$, shows that

$$
\nu(I) \leq \ell(I \cap L)+\nu(I \bar{R}),
$$

and the claim follows since $\bar{R}$ is Cohen-Macaulay, the usual bound $\nu(I \bar{R}) \leq \operatorname{deg}(\bar{R})$ holds for all ideals and

$$
\nu(I) \leq \ell(L)+\operatorname{deg}(\bar{R})=\operatorname{hdeg}(R)=e_{h}(R) .
$$

The argument also shows that, given that $e_{h}(R) \geq e_{h}(\bar{R})+\ell\left(\Gamma_{\mathfrak{m}}(R)\right)$, we may assume that $\operatorname{depth}(R) \geq 1$ in all cases.

If $d>1$, pass to the $d-1$ dimensional ring $R /\left(x^{s}\right)$. The ideal $I /\left(x^{s}\right)$ is $\mathfrak{m} /\left(x^{s}\right)-$ primary, so, by induction,

$$
\nu\left(I /\left(x^{s}\right)\right) \leq s^{d-2} \cdot e_{h}\left(\left(R /\left(x^{s}\right)\right)+d-2 .\right.
$$

Hence

$$
\nu(I) \leq \nu\left(I /\left(x^{s}\right)\right)+1 \leq s^{d-2} \cdot s \cdot e_{h}(R)+d-1,
$$

as $e_{h}\left(R /\left(x^{s}\right)\right) \leq s \cdot e_{h}(R)$ by Corollary 2.16 . 
We recall that if $I$ is an ideal, a reduction is another ideal $J \subset I$ such that $I^{n+1}=J I^{n}$ and the least such integer $n$, denoted $\operatorname{red}_{J}(I)$, is called the reduction number of $I$ relative to $J$. They are controlling elements in the theory of Rees algebras (see [15] for more details). If $(R, \mathfrak{m})$ is a Noetherian local ring with infinite residue field, the lifts of Noether normalizations of $F(I)$ give minimal reductions for $I$; the reduction number of $I$ is the minimum of all such $\operatorname{red}_{J}(I)$.

As in [8], this bound together with the main result of [5] yields:

Corollary 3.6. Let $(R, \mathfrak{m})$ be a local ring of dimension $d>0$, with infinite residue field, which is the homomorphic image of a Gorenstein ring. Then $r(\mathfrak{m}) \leq d$ ! . $e_{h}(R)-1$.

This method permits us to estimate $r(I)$ for ideals primary for the maximal ideal. A similar calculation gives that if $I$ is an $\mathfrak{m}$-primary ideal, then

$$
r(I) \leq d ! \cdot \ell(R / I)^{d-1} \cdot e_{h}(R)-1 .
$$

The presence of good depth properties in the Rees algebra of $I$ or on its special fiber $F(I)$ cuts down these estimates for the reduction of $I$. Here is one way this occurs.

Theorem 3.7. Let $(R, \mathfrak{m})$ be an analytically equidimensional local ring with residue field of characteristic zero. Let I be an $\mathfrak{m}$-primary ideal. Suppose that the Rees algebra $R[I t]$ of I satisfies the condition $S_{2}$ of Serre (e.g. $R[I t]$ is normal). Then $\operatorname{red}(I) \leq e(I ; R)-1 .\left(\operatorname{deg}\left(\operatorname{gr}_{I}(R)\right)=e(I ; R)\right.$ is the multiplicity of the ideal $\left.I.\right)$

Proof. The hypothesis implies that the associated graded ring of $I$,

$$
\operatorname{gr}_{I}(R)=R[I t] \otimes_{R}(R / I),
$$

has the condition $S_{1}$ of Serre and is equidimensional.

Since the characteristic of the residue field $R / \mathfrak{m}$ is zero, the Artinian ring $R / I$ will contain a field $k$ that maps onto the residue field $R / \mathfrak{m}$. Let $k\left[t_{1}, \ldots, t_{d}\right]$ be a standard Noether normalization of the special fiber $F(I)=\operatorname{gr}_{I}(R) \otimes(R / \mathfrak{m})$. We can lift it to $\operatorname{gr}_{I}(R)$ and therefore assume that $\operatorname{gr}_{I}(R)$ is a finitely generated, graded module over $A=k\left[t_{1}, \ldots, t_{d}\right]$ of rank equal to the multiplicity $e(I ; R)$. As all associated primes of $\operatorname{gr}_{I}(R)$ have the same dimension, we can bound reduction number by arithmetic degree (see [16]),

$$
\operatorname{red}(I)=\operatorname{red}\left(\operatorname{gr}_{I}(R)\right)<\operatorname{adeg}\left(\operatorname{gr}_{I}(R)\right)=\operatorname{deg}\left(\operatorname{gr}_{I}(R)\right)=e(I ; R),
$$

which gives the estimate.

Remark 3.8. The issue of defining $e_{h}(M)$ for modules over arbitrary Noetherian local rings and of using it as an index of regularity will be pursued elsewhere.

The author is grateful to the referee for a careful and detailed reading.

\section{REFERENCES}

1. Bayer, D., Mumford, D., What can be computed in Algebraic Geometry? D. Eisenbud et al. (eds.), Computational Algebraic Geometry and Commutative Algebra, pp. 1-48, Cortona 1991, Cambridge: Cambridge University Press, 1993. MR 95d:13032

2. Bayer, D., Stillman, M., Macaulay, a system for computation in algebraic geometry and commutative algebra, 1992. Available via anonymous ftp from zariski.harvard.edu

3. Bruns, W., Herzog, J., Cohen-Macaulay Rings, Cambridge University Press, Cambridge, 1993 MR 95h: 13020 
4. Capani, A., Niesi, G., Robbiano, L. CoCoA, a system for doing computations in commutative algebra, 1995. Available via anonymous ftp from lancelot.dima.unige.it

5. Eakin, P., Sathaye, A., Prestable ideals, J. Algebra 41 (1976), 439-454 MR 54:7449

6. Hartshorne, R., Connectedness of the Hilbert scheme, Publications Math. I.H.E.S. 29 (1966), 261-304. MR 35:4232

7. Miyazaki, C., Vogel, W., Towards a theory of arithmetic degree, Manuscripta Math. 89 (1996), 427-438. MR 97f: 14003

8. Sally, J.D., Bounds for numbers of generators for Cohen-Macaulay ideals, Pacific J. Math. 63 (1976), 517-520. MR 53:13208

9. Sally. J.D., Numbers of Generators of Ideals in Local Rings, Lecture Notes in Pure \& Applied Math. 35. Marcel Dekker, New York, 1978. MR 58:5654

10. Stückrad, J., Vogel, W., Buchsbaum Rings and Applications, Springer, Vienna-New York, 1986. MR 88h:13011

11. Sturmfels, B., Trung, N.V., Vogel, W., Bounds on degrees of projective schemes, Math. Annalen 302 (1995), 417-432. MR 96i:13029

12. Ngo Viet Trung, Reduction exponent and degree bound for the defining equations of graded rings, Proc. Amer. Math. Soc. 101 (1987), 229-236. MR 89i:13031

13. Ngo Viet Trung, Bounds for the minimum number of generators of generalized CohenMacaulay ideals, J. Algebra 90 (1984), 1-9. MR 85h:13012

14. Valla, G., Generators of ideals and multiplicities, Comm. in Algebra 9 (1981), 1541-1549. MR 83d:13030

15. Vasconcelos, W.V., Arithmetic of Blowup Algebras, London Math. Soc., Lecture Note Series 195, Cambridge University Press, Cambridge, 1994. MR 95g:13005

16. Vasconcelos, W.V., The reduction number of an algebra, Compositio Math. 104 (1996), 189-197. CMP 97:04

Department of Mathematics - Hill Center, Rutgers University, 110 Frelinghuysen RD, Piscataway, New Jersey 08854-8019

E-mail address: vasconce@math.rutgers.edu 